\title{
Linear and Weakly Nonlinear Analyses of Magneto- Convection in a Sparsely Packed Porous Medium under Gravity Modulation
}

\author{
R. Ragoju ${ }^{\dagger}$ and S. Shekhar \\ Department of Applied Sciences, National Institute of Technology Goa, Goa 403401, India \\ † Corresponding Author Email: ravi@nitgoa.ac.in
}

(Received March 3, 2020; accepted May 25, 2020)

\begin{abstract}
This paper deals with the linear stability analysis and weakly non-linear analysis of Magneto-convection in a sparsely packed porous medium with constant vertical Magnetic field and gravity modulation. A linear stability analysis reported here and shows that the gravity modulation has significant effect on the stability limits of the system. The gravity modulation is know to have effect and is treated by a perturbation expansion in powers of the amplitude of modulation. The shift in the critical Rayleigh number is evaluated and depends on the prandtl number and frequency of modulation, using the Venezian method. It is also shown that the onset of convection can be advance or delay by the regulation of various parameters. Weakly nonlinear analysis is performed based on the method of power series, where the disturbance is expressed in terms of power series. A nonlinear Ginzburg-Landau equation to investigate the three different types of gravity modulation on heat transfer is derived as part of this work. Heat transfer have been shown to depend on Nusselt number, further the effect of different types of parameter on heat transport have been studied graphically. Nusselt number graph is also shown for different parameter and explain in detail. The effect of magnetic prandtl number and Chandrasekhar number are stabilize the system. The control of convection is a major issue in systems with fluids as a working media. This is all the more difficult if the fluid system is housed in a porous medium. The paper presents three mechanisms of controlling onset of convection and thereby the heat transfer in such fluid systems. In order the modulation effect is effective in its role, we have considered the system to be a fluid-saturated porous media.
\end{abstract}

Keywords: Magneto-convection; Sparsely packed porous medium; Ginzburg-Landau equation; Gravity modulation; Heat transfer.

\section{NOMENCLATURE}

magnetic potential amplitude of streamline perturbation height of fluid liquid

Darcy number

acceleration due to gravity magnetic field permeability of porous medium dimensionless heat capacity Nusselt number pressure thermal diffusivity Prandtl number magnetic prandtl number chandrasekhar number Rayleigh number time temperature temperature difference mean flow velocity space co-ordinates $\alpha \quad$ coefficient of thermal expansion

$\mu_{e} \quad$ coefficient of effective fluid viscosity

$\mu_{m} \quad$ magnetic permeability

$\mu \quad$ coefficient of viscosity of fluid

$\phi \quad$ porosity

$\eta \quad$ magnetic diffusivity

$\rho$ density

$\varepsilon \quad$ perturbation parameter

$\omega$ frequency of modulation

$\delta_{1} \quad$ amplitude of modulation

$\sigma \quad$ growth rate

$\psi \quad$ stream function

Superscripts

Subscripts

perturbated quantity

b base state

c critical value

0 reference valve 


\section{INTRODUCTION}

Magneto-convection with a sparsely packed porous medium is heated from the bottom, uniformly, is very interesting phenomenon in fluid dynamics specially in geophysics. This event may happen inside the layer of the Earth's mushy outer core. The peripheral core of earth consists of mostly Iron, sulphur and Nickel which is in fluid layer about 2400 K.M thick, the temperature of outer core is about $3000 \mathrm{~K}-4500 \mathrm{~K}$, iron-nickel fluid of the outer core is main source of Earth's magnetic fluid. At the surface of the earth the magnetic field is 50 times less stronger than at the core. In an electrically conducting liquid in non-porous case, magnetoconvection has been studied by many researcher in detail but, in sparsely porous medium it has not been studied in this way, and it has many application in geophysical fluid dynamics.

Gershuni (1970) and Gresho et al. (1970) have studied the stability analysis under the presence of the gravity modulation on a fluid layer heated from below. The result confirms that the layer being heated from below under gravity modulation is stable, but the layer is destabilized in the case of heating from above. Clever et al. (1993) have studied the two-dimensional modes of oscillating convection of fluid layer for free-free and rigid-rigid boundaries heated either from above or from below, under the gravity modulation and concluded that the subharmonic modes are unstable for the finiteamplitude synchronous convection. The low frequency g-jitter are known to affect the stability of the system and viscous flow limit and the Darcy limit are the degenerate cases of the Brinkman model and also the asymptotic analysis are discussed for various frequencies.

Malashetty and Padmavathi (1997) have studied in a fluid the small amplitude onset of convection and its effects, and concluded that low frequency g-jitter definitely affects the stability of the fluid layers. Dyko and Vafai (2007) have discussed about the convection in two horizontal coaxial cylinders in presence of gravity modulation and their result shows that gravity modulation has stabilized the secondary flow as compared to gravity with constant force. Eckhaus and zigzag instabilities in thermal convection of couple stress fluid under the influence of Soret and Dufour effects were reported by Ravi et al. (2018) and also the heat and mass transports for unmodulated case were discussed. Malashetty and swamy (2011) examined, in viscous fluids the thermal convection of porous layer with gravitational modulation in influence of the rotation and made a conclusion that gravity modulation provides stabilizing effect and in Brinkman porous layer both stabilizing and destabilizing effects. Siddheshwar $e t$ al. (2011), have reported the stationary magnetoconvection in the presence of newtonian liquid and concluded that the system is stabilized by magnetic field. Bhadauria et al. (2013) studied the combined effect of both gravity modulation and temperature and internal heating in a medium which is porous and closely packed and heated from below and same time cooled from above and observed that gravity modulation results in oscillatory movements in the system, transport of heat in internal parts is more than when internal heating is not present.

Malashetty and Begum (2011), in their paper explained that gravity modulation stabilizes as well as destabilizes saturated porous medium for a Maxwell fluid and it depends on the various parameters like frequency and many more. Bhadauria et al. (2012) have studied the effect of time-periodic temperature/gravity modulation on the thermal instability. Bhadauria et al. (2013) have reported that the combined effect due to variable viscosity and modulation of temperature in the anisotropic porous medium. A weakly non-linear theory performed to investigate the heat transport in presence of temperature modulation and made a conclusion that heat transport is negligible in the system for In-phase modulation. Many researchers like, Bhadauria and Khan (2009), Siddheswar (2010), Shivakumara et al. (2011), Babu et al. (2011), Bhadauria (2016) have explain in detail for the effect of temperature modulation and gravity modulation in different situation under porous medium. Farooq et al. (1996), investigated convection under gravity modulation for a vertical slot and concluded that gravity modulation destabilize the long-wave eigenmodes of the slot problem for $\varepsilon \sim \mathrm{O}(1)$.

The proposed work is presented as follows: The relevant governing equation with perturbation is discussed in section 2 followed by section 3 with linear stability analysis, and the finite amplitude equation, weakly nonlinear stability analysis using different types of gravity modulation and heat transport is described in section 4. The results and discussions are presented in section 5. And concluding remarks of the presented work are in section 6 .

\section{GOVERNING EQUATIONS}

We consider an electrically and thermally conducting fluid in sparely packed porous medium of depth $d$, confined between two infinitely, parallel, horizontal layer at $z=0$ and $z=d$ with uniform magnetic field $\mathrm{H}_{0}$ in a vertical direction given in Fig. 1. The magneto-convection with sparsely packed porous medium under boussinesq approximation, the set of governing equations are (Babu et al. 2011):

$\nabla \cdot \vec{V}=0$

$\nabla \cdot \vec{H}=0$

$\rho_{0}\left[\frac{1}{\phi} \frac{\partial \vec{V}}{\partial t}+\frac{1}{\phi^{2}}(\vec{V} . \nabla) \vec{V}\right]-\frac{\mu_{m}}{4 \pi}\left[H_{0} \frac{\partial \vec{H}}{\partial z}+(\vec{H} . \nabla) \vec{H}\right]$

$=-\nabla\left[P+\frac{\mu}{8 \pi}|\vec{H}|^{2}+\frac{\mu_{m} H_{0}}{4 \pi^{2}} H_{z}\right]+\rho g-\frac{\mu}{K} \vec{V}$

$+\mu_{e} \nabla^{2} \vec{V}$

$\mathrm{M} \frac{\partial \mathrm{T}}{\partial \mathrm{t}}+(\overrightarrow{\mathrm{V}} \cdot \nabla) \mathrm{T}=\mathrm{k}_{\mathrm{T}} \nabla^{2} \overrightarrow{\mathrm{T}}$,

$\varphi \cdot \frac{\partial \overrightarrow{\mathrm{H}}}{\partial \mathrm{t}}=\nabla \times\left(\overrightarrow{\mathrm{V}} \times \mathrm{H}_{0} \hat{\mathrm{e}}_{\mathrm{z}}\right)+\nabla \times(\overrightarrow{\mathrm{V}} \times \overrightarrow{\mathrm{H}})+\eta \nabla^{2} \overrightarrow{\mathrm{H}}$, 


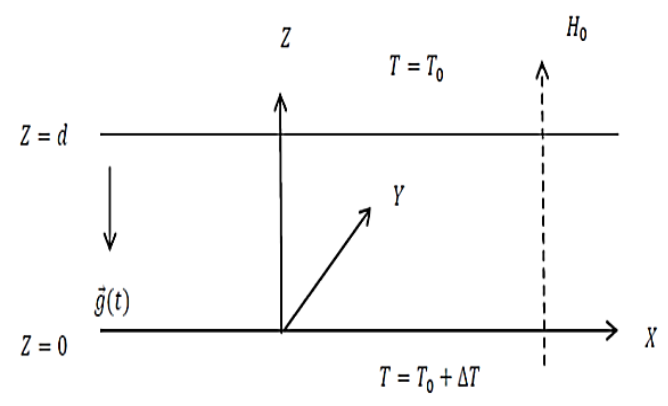

Fig. 1. Physical configuration of problem.

$$
\begin{aligned}
& \rho=\rho_{0}\left[1-\alpha\left(\mathrm{T}-\mathrm{T}_{0}\right)\right], \\
& g(t)=g_{0}\left[1+\varepsilon^{2} \delta_{1} \sin (\omega t)\right] \hat{k} .
\end{aligned}
$$

All quantities which are used in the above equations have explained in nomenclature. The thermal boundary conditions are as follows:

$\mathrm{T}=\mathrm{T}_{0}+\Delta \mathrm{T}$, at $\mathrm{z}=0, \mathrm{~T}=\mathrm{T}_{0}$, at $\mathrm{z}=\mathrm{d}$.

We assume that the basic state quiescent. The basic state solutions are given by:

$$
\mathrm{V}_{\mathrm{b}}=0, \rho=\rho_{\mathrm{b}}(\mathrm{z}), \mathrm{T}=\mathrm{T}_{\mathrm{b}}(\mathrm{z}), \mathrm{P}=\mathrm{P}_{\mathrm{b}}(\mathrm{z})
$$

using Eq. (8) the expression of basic state temperature is:

$T_{b}=T_{b}+\Delta T\left(1-\frac{z}{d}\right)$

Now we introduced the finite-amplitude perturbations on basic solution:

$\overrightarrow{\mathrm{V}}=\overrightarrow{\mathrm{V}}^{\prime}+\overrightarrow{\mathrm{V}}_{\mathrm{b}}, \mathrm{T}=\mathrm{T}^{\prime}+\mathrm{T}_{\mathrm{b}}(\mathrm{z}), \mathrm{P}=\mathrm{P}^{\prime}+\mathrm{P}_{\mathrm{b}}(\mathrm{z})$

$\overrightarrow{\mathrm{H}}=\overrightarrow{\mathrm{H}}^{\prime}+\mathrm{H}_{\mathrm{b}}(\mathrm{z}), \rho=\rho^{\prime}+\rho_{\mathrm{b}}(\mathrm{z})$.

Further, we consider only disturbances in two direction ( $x$-direction and $z$-direction) in our study, now using Eqs. (11) and (12) into Eqs. (1)-(6), and introducing stream function $\psi$ as $(\mathrm{u}, 0, \mathrm{w})=\left(\frac{\partial \psi}{\partial \mathrm{z}}, 0,-\frac{\partial \psi}{\partial \mathrm{x}}\right)$ and magnetic potential $\mathrm{A}$ as $\left(\mathrm{H}_{1}, \mathrm{H}_{2}, \mathrm{H}_{3}\right)=\left(\frac{\partial \mathrm{A}}{\partial \mathrm{z}}, 0,-\frac{\partial \mathrm{A}}{\partial \mathrm{x}}\right)$ Resulting system of equations are non-dimensionalized as for the following scales:

$$
\begin{aligned}
& (\mathrm{x}, 0, \mathrm{z})=\left(\frac{\mathrm{x}^{\prime}}{\mathrm{d}}, 0, \frac{\mathrm{z}^{\prime}}{\mathrm{d}}\right), \tau=\frac{\mathrm{t}^{\prime}}{\mathrm{Md}^{2} / \mathrm{k}_{\mathrm{T}}}, \mathrm{u}=\frac{\mathrm{u}^{\prime}}{\mathrm{k}_{\mathrm{T}} / \mathrm{Md}}, \\
& \mathrm{w}=\frac{\mathrm{w}^{\prime}}{\mathrm{k}_{\mathrm{T}} / \mathrm{Md}}, \mathrm{T}=\frac{\mathrm{T}^{\prime}}{\Delta \mathrm{T}}, \mathrm{P}=\frac{\mathrm{P}^{\prime}}{\rho_{0} \mathrm{k}_{\mathrm{T}}^{2} \mathrm{M}^{-2} \mathrm{~d}^{-2}}, \\
& \mathrm{H}=\frac{\mathrm{H}^{\prime}}{\mathrm{k}_{\mathrm{T}} \mathrm{H}_{0} / \eta}, \psi=\frac{\psi^{\prime}}{\mathrm{k}_{\mathrm{T}} / \mathrm{M}}, \mathrm{A}=\frac{\mathrm{A}^{\prime}}{\mathrm{k}_{\mathrm{T}} \mathrm{H}_{0} \mathrm{~d} / \eta} .
\end{aligned}
$$

Now for simplicity drop the asterisk then we get the equations as:

$$
\begin{aligned}
& \frac{1}{M D a}\left(\nabla^{2} \psi\right)-\frac{\Lambda}{M} \nabla^{4} \psi+\left(1+g_{m}\right) R a \frac{\partial T}{\partial x}-Q \frac{\partial \nabla^{2} A}{\partial z} \\
& =-\frac{1}{M^{2} \phi P r_{1}} \frac{\partial \nabla^{2} \psi}{\partial \tau}+\frac{1}{M^{2} \phi^{2} P r_{1}} \frac{\partial\left(\psi, \nabla^{2} \psi\right)}{\partial(\mathrm{x}, \mathrm{z})} \\
& -Q \frac{\operatorname{Pr}_{2}}{P r_{1}} \frac{\partial\left(A, \nabla^{2} A\right)}{\partial(x, z)}
\end{aligned}
$$

$\frac{1}{M}-\frac{\partial \psi}{\partial x}-\nabla^{2} T=\frac{\partial T}{\partial \tau}+\frac{1}{M} \frac{\partial(\psi, T)}{\partial(x, z)}$

$-\frac{\partial \psi}{\partial z}-M \nabla^{2} A=-\phi \frac{P r_{2}}{P r_{1}} \frac{\partial A}{\partial \tau}+\frac{P r_{2}}{P r_{1}} \frac{\partial(\psi, A)}{\partial(x, z)}$

Where $\vartheta=\frac{\mu}{\rho_{0}}, \operatorname{Pr}_{1}=\frac{\vartheta}{k_{T}}, \operatorname{Pr}_{2}=\frac{\vartheta}{\eta}, \Lambda=\frac{\mu_{e}}{\mu}$ $Q=\frac{\mu_{m} H_{0}^{2} d^{2}}{4 \pi \rho_{0} \vartheta \eta}, D a=\frac{K}{d^{2}}, R a=\frac{\alpha g_{0} \Delta T d^{3}}{k_{T} \vartheta}$

The non-linear Eqs. (13)-(15) can be represented using the matrices is as follows:

$$
\begin{gathered}
{\left[\begin{array}{ccc}
\left.\frac{\nabla^{2}}{M D a}-\frac{\Lambda \nabla^{4}}{M}\right) & \left(1+g_{m}\right) R_{0 c} \frac{\partial}{\partial x} & -Q \frac{\partial}{\partial z} \nabla^{2} \\
\frac{1}{M} \frac{\partial}{\partial x} & -\nabla^{2} & 0 \\
-\frac{\partial}{\partial z} & 0 & -M \nabla^{2}
\end{array}\right]\left[\begin{array}{c}
\psi \\
T \\
A
\end{array}\right]} \\
=\left[\begin{array}{c}
\frac{1}{M^{2} \phi P r_{1}} \frac{\partial \nabla^{2} \psi}{\partial \tau}+\frac{1}{M^{2} \phi^{2} P r_{1}} \frac{\partial\left(\psi, \nabla^{2}\right)}{\partial(x, z)}-Q \frac{P r_{2}}{P r_{1}} \frac{\partial\left(A, \nabla^{2} A\right)}{\partial(x, z)} \\
-\frac{\partial T}{\partial \tau}+\frac{1}{M} \frac{\partial(\psi, T)}{\partial(x, z)} \\
-\phi \frac{P r_{2}}{P r_{1}} \frac{\partial A}{\partial \tau}+\frac{P r_{2}}{P r_{1}} \frac{\partial(\psi, A)}{\partial(x, z)}
\end{array}\right]
\end{gathered}
$$

case(1): Trigonometry wave-form

$$
g_{m}(\omega, \tau)=\varepsilon^{2} \delta_{1} \sin (\omega \tau)
$$

case(2): Triangular wave-form

$g_{m}(\omega, \tau)=\varepsilon^{2} \frac{8 \delta_{1}}{\pi^{2}} \sum_{n=1,3,5, \ldots}^{\infty} \frac{(-1)^{\left(\frac{n-1}{2}\right)}}{n^{2}} \sin (n \omega \tau)$

case(3): Square wave-form 
R. Ragoju and S. Shekhar / JAFM, Vol. 13, No. 6, pp. 1937-1947, 2020.

$$
g_{m}(\omega, \tau)=\varepsilon^{2} \frac{4 \delta_{1}}{\pi} \sum_{n=1,3,5, \ldots}^{\infty} \frac{-1}{n} \sin (n \omega \tau)
$$

Derivation of Amplitude equation for above three different gravity modulation cases were discussed in section 4.

\section{LINEAR STABILITY ANALYSIS}

The jacobin terms present in the Eqs. (13), (14) and (15) are neglected to study the linear stability.

$$
\begin{gathered}
\frac{1}{M^{2} \phi \operatorname{Pr}_{1}} \frac{\partial \psi}{\partial \tau} \nabla^{2}-Q \frac{\partial \nabla^{2} A}{\partial z}=-\frac{1}{M D a} \nabla^{2} \psi \\
-g_{m} R a \frac{\partial T}{\partial x}+\frac{\Lambda}{M} \nabla^{4} \psi \\
\frac{\partial T}{\partial \tau}-\nabla^{2} T=-\frac{1}{M} \frac{\partial \psi}{\partial x} \\
\phi \frac{\operatorname{Pr}_{2}}{\operatorname{Pr}_{1}} \frac{\partial A}{\partial \tau}-M \nabla^{2} A=\frac{\partial \psi}{\partial z}
\end{gathered}
$$

Eliminating $\mathrm{T}$ and A from the above Eqs. (19), (20) and (21), so we resulting equation as follows,

$$
\begin{gathered}
\frac{1}{M D a}\left(\frac{\partial}{\partial \tau}-\nabla^{2}\right)\left(\phi \frac{P r_{2}}{P r_{1}} \frac{\partial}{\partial \tau}-M \nabla^{2}\right) \nabla^{2} \psi \\
+\frac{\Lambda}{M}\left(\frac{\partial}{\partial \tau}-\nabla^{2}\right)\left(\phi \frac{P r_{2}}{P r_{1}} \frac{\partial}{\partial \tau}-M \nabla^{2}\right) \nabla^{4} \psi \\
-\frac{g_{m} R_{a}}{M}\left(\phi \frac{P r_{2}}{P r_{1}} \frac{\partial}{\partial \tau}-M \nabla^{2}\right) \frac{\partial^{2} \psi}{\partial x^{2}} \\
+Q\left(\frac{\partial}{\partial \tau}-\nabla^{2}\right) \frac{\partial^{2}}{\partial z^{2}} \nabla^{2} \psi \\
-\left(\frac{1}{M_{2} \phi P r_{1}} \frac{\partial}{\partial \tau}\right)\left(\frac{\partial}{\partial \tau}-\nabla^{2}\right)\left(\phi \frac{P r_{2}}{P r_{1}} \frac{\partial}{\partial \tau}-M \nabla^{2}\right) \nabla^{2} \psi=0
\end{gathered}
$$

$\psi$ is satisfied the boundary condition and $\psi=\nabla^{2} \psi=\nabla^{4} \psi=\nabla^{6} \psi=0$ at $z=0,1$.

Now we introduce the asymptotic expansions as,

$$
\begin{aligned}
& R a=R_{0 c}+\varepsilon R_{1}+\varepsilon^{2} R_{2}+\ldots \\
& \psi=\psi_{0}+\varepsilon \psi_{1}+\varepsilon^{2} \psi_{2}+\ldots
\end{aligned}
$$

Here $\mathrm{R}_{0 c}$ is eigenvalue and $\psi_{0}$ is eigenfunction for unmodulated case and $\mathrm{Ri}$ and $\psi_{i}$, for $i \geq 1$ for modulated case. Now using Eqs. (23) and (24) in Eq.(22) and collect the coefficient of various power of $\varepsilon$ from resulting equation we get:

$$
L \psi_{0}=0
$$

$$
L \psi_{1}=\phi \frac{P r_{2}}{P r_{1}} k^{2}\left[R_{0} f(t)+R_{1}\right] \nabla^{2} \psi_{0}
$$

$$
L \psi_{2}=\phi \frac{P r_{2}}{P r_{1}} k^{2}\left[R_{0} f(t) \nabla^{2} \psi_{1}+R_{2} \nabla^{2} \psi_{0}\right]
$$

where,

$$
\begin{gathered}
L=\left(\frac{\partial}{\partial z}-\nabla^{2}\right)\left(\phi \frac{P r_{2}}{P r_{1}} \frac{\partial}{\partial \tau}-M \nabla^{2}\right) \frac{1}{M D a} \nabla^{2} \\
+\frac{\Lambda}{M}\left(\frac{\partial}{\partial \tau}-\nabla^{2}\right)\left(\phi \frac{P r_{2}}{P r_{1}} \frac{\partial}{\partial \tau}-M \nabla^{2}\right) \nabla^{4} \\
-\frac{R_{0}}{M}\left(\phi \frac{P r_{2}}{P r_{1}} \frac{\partial}{\partial \tau}-M \nabla^{2}\right) \frac{\partial^{2}}{\partial x^{2}} \\
+\left(\frac{\partial}{\partial z}-\nabla^{2}\right) Q \frac{\partial^{2}}{\partial z^{2}} \nabla^{2} \\
-\frac{1}{M_{2} \phi P r_{1}} \frac{\partial}{\partial \tau}\left(\frac{\partial}{\partial \tau}-\nabla^{2}\right)\left(\phi \frac{P r_{2}}{P r_{1}} \frac{\partial}{\partial \tau}-M \nabla^{2}\right) \nabla^{2}
\end{gathered}
$$

solution of zero order problem let

$\psi_{0}=\sin (k x) \sin (\pi z)$

putting the value of Eq. (28) in Eq. (25) we get,

$R_{0 c}=\frac{\delta^{2}\left[Q \pi^{2}+\delta^{2}\left(\frac{1}{D a}+\Lambda \delta^{2}\right)\right]}{k^{2}}$

where, $\delta^{2}=k^{2}+\pi^{2}$

now Eq. (26) using Eq. (28) becomes

$L \psi_{1}=-\delta^{2} \phi \frac{P r_{2}}{P r_{1}} k^{2}\left[R_{0} f(t) \sin (\pi z)+R_{1} \sin (\pi z)\right]$

For the solution of above equation have to exist, the null-space operator L must be orthogonal to the right-hand side of the above equation. It means that the rhs of the time-independent equation must be orthogonal to $\sin (\pi z)$, that is $R_{l}=0$. The steady term on rhs of the Eq. (30) is $R o f(t) \sin (\pi z)$, since $f$ changing sinusoidal with time. Then it is clear that all the odds coefficients $R_{1}=R_{3}=R_{5}=0$. We find that

$$
L\left(\sin \pi z e^{i(k x-\omega t)}\right)=L(\omega) \sin \pi z e^{i(k x-\omega t)}
$$

where,

$$
\begin{aligned}
& L(\omega)=X_{1}+i X_{2} \\
& X_{1}=\phi \frac{P r_{2}}{\operatorname{Pr}_{1}} \frac{\omega^{2} \delta^{2}}{M D a}-\frac{\delta^{6}}{D a}-\frac{\omega^{2} M \delta^{4}}{M^{2} \phi P r_{1}}-\frac{P r_{2}}{P r_{1}} \frac{\omega^{2} \delta^{4}}{M^{2} P r_{1}} \\
& -\frac{P r_{2}}{P r_{1}} \frac{\omega^{2} \Lambda \delta^{4}}{M}+\Lambda \delta^{8}+R_{0} \delta^{2} k^{2}+Q \delta^{4} \pi^{2} \\
& X_{2}=\omega\left[\frac{P r_{2}}{\operatorname{Pr}_{1}} \frac{\omega^{3} \delta^{2}}{M^{2} D a}-\frac{\delta^{6}}{M \phi P r_{1}}+\frac{\delta^{4}}{D a}-\frac{P r_{2}}{P r_{1}} \frac{\phi \delta^{4}}{M D a}\right] \\
& -\omega\left[\Lambda \delta^{6}-\frac{\operatorname{Pr}_{2}}{\operatorname{Pr}_{1}} \frac{\phi \Lambda \delta^{6}}{M}-Q \delta^{2} \pi^{2}\right] \\
& \delta^{2}=k^{2}+\pi^{2}
\end{aligned}
$$

the particular solution of Eq. (30) is

$$
\begin{aligned}
& \psi_{1}=\frac{1}{|L(\omega)|^{2}} \phi\left(\frac{P r_{2}}{P r_{1}}\right)\left(X_{1} \cos \omega t-X_{2} \sin \omega t\right) \\
& \left(-R_{0} k^{2} \sin \pi z\right)
\end{aligned}
$$


R. Ragoju and S. Shekhar / JAFM, Vol. 13, No. 6, pp. 1937-1947, 2020.

The solution of Eq. (30) for corresponding homogeneous part has a term which is proportional to $\sin \pi \mathrm{z}$, adding a similar term in Eq. (30) to make complete solution, re-normalize the term because we can group all the terms proportional to $\sin \pi \mathrm{z}$ and define a $\psi_{0}$ with corresponding $\psi_{1}, \psi_{2}, \psi_{3}, \ldots .$. so we let that $\psi_{0}$ is orthogonal to all others $\psi_{\mathrm{n}}^{\prime} \mathrm{s}$. The equation of $\psi_{2}$ can be as

$$
L \psi_{2}=\phi \frac{P r_{2}}{P r_{1}} k^{2}\left[R_{0} f(t) \nabla^{2} \psi_{1}+R_{2} \nabla^{2} \psi_{0}\right]
$$

Here we shall not require the solution of the Eq.(33), but we use this to find $\mathrm{R}_{2}$. For the solution of the Eq. (33) have to exist, its rhs steady part must be orthogonal to $\sin \pi z$. This gives us,

$$
\begin{array}{r}
-\int_{0}^{1} \phi \frac{P r_{2}}{P_{1}} k^{2}\left[R_{0} f(t) \delta^{2} \psi_{1}+R_{2} \delta^{2} \psi_{0}\right] \\
\sin (\pi z) d z=0
\end{array}
$$

Taking time average, we get,

$$
R_{2}=R_{0}^{2} \int_{0}^{1} \phi \frac{P r_{2}}{P r_{1}} k^{2} \delta^{2} \overline{\left[f(t) \psi_{1}\right]} \sin (\pi z) d z
$$

Following Venezian (1969), we get the expression for $\mathrm{R}_{2}$ in the form

$$
R_{2}=R_{0}^{2}\left(\frac{P r_{2}}{P r_{1}} \phi k^{2} \delta^{2} \frac{X_{1}}{2|L(\omega)|^{2}}\right)
$$

\section{FINITE AMPLITUDE EQUATION}

We introduce the following power series expansion interms of $\varepsilon$ (Venezian (1969), Malkus (1958)).

$$
\begin{aligned}
& R a=R_{0 c}+\varepsilon^{2} R_{2}+\varepsilon^{4} R_{4}+\varepsilon^{6} R_{6}+\ldots \\
& \psi=\varepsilon \psi_{1}+\varepsilon^{2} \psi_{2}+\varepsilon^{3} \psi_{3}+\ldots \\
& T=\varepsilon T_{1}+\varepsilon^{2} T_{2}+\varepsilon^{3} T_{3}+\ldots \\
& A=\varepsilon A_{1}+\varepsilon^{2} A_{2}+\varepsilon^{3} A_{3}+\ldots
\end{aligned}
$$

We use the small-time variations, for that we rescaling as $\tau=\varepsilon^{2} t$.

Now using Eqs. (37)-(40) in Eqs. (13)-(15) and collect the coefficient of the lowest order of $\varepsilon$, we get the following system:

$$
\begin{aligned}
& {\left[\begin{array}{ccc}
\left(\frac{\nabla^{2}}{M D a}-\frac{\Lambda \nabla^{4}}{M}\right) & R_{0 c} \frac{\partial}{\partial x} & -Q \frac{\partial}{\partial z} \nabla^{2} \\
\frac{1}{M} \frac{\partial}{\partial x} & -\nabla^{2} & 0 \\
-\frac{\partial}{\partial z} & 0 & -M \nabla^{2}
\end{array}\right]\left[\begin{array}{l}
\psi_{1} \\
T_{1} \\
A_{1}
\end{array}\right]} \\
& =\left[\begin{array}{l}
0 \\
0 \\
0
\end{array}\right]
\end{aligned}
$$

with the conditions on the boundary as: $\psi=\nabla^{2} \psi=0$, $A=0, T=0$, on $z=0$ and $z=1$.

The first order solution of system:

$\psi_{1}=B(\tau) \sin \left(k_{c} x\right) \sin (\pi z)$

$T_{1}=\frac{-k_{c}}{M \delta^{2}} B(\tau) \cos \left(k_{c} x\right) \sin (\pi z)$

$A_{1}=\frac{\pi}{M \delta^{2}} B(\tau) \sin \left(k_{c} x\right) \cos (\pi z)$

where $\delta^{2}=k_{c}^{2}+\pi^{2}$,

the critical Rayleigh number for onset of the stationary magneto-convection can be obtained from Eq. (41)

$R_{0 c}=\frac{\delta^{2}\left[Q \pi^{2}+\delta^{2}\left(\frac{1}{D a}+\Lambda \delta^{2}\right)\right]}{k_{c}^{2}}$

By putting Chandrasekhar number $\mathrm{Q}=0$, Darcy number Da tends to infinite and $\Lambda=1$, in Eq. (45), we get classical results of critical Rayleigh number $\mathrm{R}_{\mathrm{oc}}$ of Rayleigh-Benard convection obtained by Chandrasekhar (1961).

$R_{0 c}=\frac{\delta^{6}}{k_{c}^{2}}$

Now for second order system, we get:

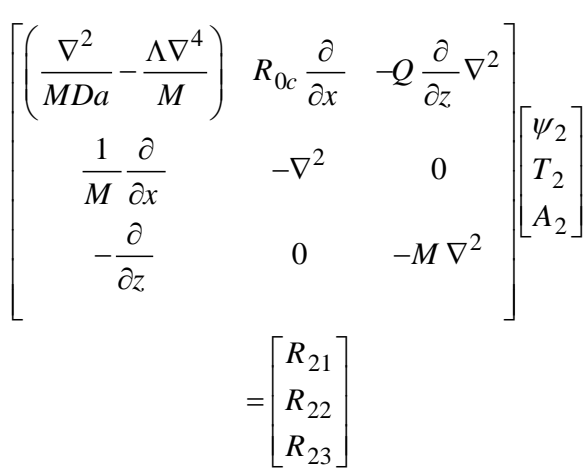

where,

$R_{21}=0$

$R_{22}=\frac{1}{M} \frac{\partial \psi_{1}}{\partial x} \frac{\partial T_{1}}{\partial z}-\frac{1}{M} \frac{\partial \psi_{1}}{\partial z} \frac{\partial T_{1}}{\partial x}$

$R_{23}=\frac{P r_{2}}{P r_{1}} \frac{\partial \psi_{1}}{\partial x} \frac{\partial A_{1}}{\partial z}-\frac{P r_{2}}{P r_{1}} \frac{\partial \psi_{1}}{\partial z} \frac{\partial A_{1}}{\partial x}$

with the boundary conditions $\psi=\nabla^{2} \psi=0, T=0, A$ $=0$ on $z=0$ and $z=1$.

The solution for second-order system, can be obtained as follows:

$\psi_{2}=0$

$T_{2}=\frac{-k_{c}^{2}}{8 M^{2} \delta^{2} \pi}[B(\tau)]^{2} \sin (2 \pi z)$ 
R. Ragoju and S. Shekhar / JAFM, Vol. 13, No. 6, pp. 1937-1947, 2020.

$$
A_{2}=-\frac{P r_{2}}{P r_{1}} \frac{\pi^{2}}{8 M^{2} \delta^{2} k_{c}}[B(\tau)]^{2} \sin \left(2 k_{c} x\right)
$$

Now for the third order system, we have

$$
\begin{aligned}
& {\left[\begin{array}{ccc}
\left(\frac{\nabla^{2}}{M D a}-\frac{\Lambda \nabla^{4}}{M}\right) & R_{0 c} \frac{\partial}{\partial x} & -Q \frac{\partial}{\partial z} \nabla^{2} \\
\frac{1}{M} \frac{\partial}{\partial x} & -\nabla^{2} & 0 \\
-\frac{\partial}{\partial z} & 0 & -M \nabla^{2}
\end{array}\right]\left[\begin{array}{l}
\psi_{3} \\
T_{3} \\
A_{3}
\end{array}\right]} \\
& =\left[\begin{array}{l}
R_{31} \\
R_{32} \\
R_{33}
\end{array}\right] \\
& R_{31}=-\frac{1}{M^{2} \phi P r_{1}} \frac{\partial \nabla^{2} \psi_{1}}{\partial \tau}+\left(R_{2}+\delta_{1} \sin (\omega t) R_{0 c}\right) \frac{\partial T_{1}}{\partial x} \\
& -\mathrm{Q} \frac{P r_{2}}{P r_{1}}\left(\frac{\partial A_{1}}{\partial \mathrm{x}} \frac{\partial \nabla^{2} A_{2}}{\partial \mathrm{z}}-\frac{\partial A_{1}}{\partial \mathrm{z}} \frac{\partial \nabla^{2} A_{2}}{\partial \mathrm{x}}\right) \\
& R_{32}=-\frac{\partial T_{1}}{\partial \tau}+\frac{1}{M} \frac{\partial \psi_{1}}{\partial x} \frac{\partial T_{2}}{\partial z} \\
& R_{33}=-\phi \frac{P r_{2}}{P r_{1}} \frac{\partial A_{1}}{\partial \tau}-\frac{P r_{2}}{P r_{1}} \frac{\partial \psi_{1}}{\partial z} \frac{\partial A_{2}}{\partial x}
\end{aligned}
$$

Now for the existence of the solution of the thirdorder system, we have used the solvability condition (Siddheshwar et al. 2011) to obtain GinzburgLandau equation in the following form of

$$
B_{1}=\frac{d B(\tau)}{d \tau}=B_{2} B(\tau)+B_{3}[B(\tau)]^{3}
$$

where,

$$
\begin{array}{r}
B_{1}=\frac{\delta^{2}}{M^{2} \phi P r_{1}}+\frac{R_{0} k_{c}^{2}}{M^{2} \delta^{4}}-Q \phi\left(\frac{P r_{2}}{P r_{1}}\right) \frac{\pi^{2}}{M^{2} \delta^{4}} \\
B_{2}=\frac{k_{c}^{2}}{M \delta^{2}}\left[R_{2}+R_{0} \delta_{1} \sin (\omega \tau)\right] \\
B_{3}=Q\left(\frac{P r_{2}}{P r_{1}}\right)^{2} \frac{\pi^{4}}{2 M^{3} \delta^{2}}\left[\frac{1}{4}-\frac{k_{c}^{2}}{\delta^{2}}\right]-\frac{R_{0} k_{c}^{4}}{8 M^{4} \delta^{4}} \\
+Q\left(\frac{P r_{2}}{P r_{1}}\right)^{2} \frac{\pi^{6}}{8 M^{3} \delta^{4}}
\end{array}
$$

The Ginzburg-Landau equation given in Eq. (56) is Bernoulli equation, and since the equation is of nonautonomous nature, it is very difficult to find its analytical solution, therefore it has been solved numerically. The solution of Eq. (56) can be found by using the in-built function ode45 MATLAB $\mathrm{R} 2020 \mathrm{a}$ subject to the initial condition $B(0)=c_{0}$ where $c_{0}$ is an initial amplitude of convection, for simplicity we may assume $\mathrm{R}_{2}=\mathrm{R}_{0 c}$ to keep the parameters to be minimum.
Now in similar manner, we write Ginzburg-Landau equation for case (2) and case (3) as

$$
\begin{aligned}
& B_{1} \frac{d B(\tau)}{d \tau}=\frac{k_{c}^{2}}{M \delta^{2}} \\
& {\left[R_{2}+R_{0} \frac{8 \delta_{1}}{\pi^{2}} \sum_{n=1,3,5, \ldots}^{\infty} \frac{(-1)\left(\frac{n-1}{2}\right)}{n^{2}} \sin (n \omega \tau)\right] B(\tau)} \\
& +B_{3}[B(\tau)]^{3} \\
& B_{1} \frac{d B(\tau)}{d \tau}=\frac{k_{c}^{2}}{M \delta^{2}} \\
& {\left[R_{2}+R_{0} \frac{4 \delta_{1}}{\pi} \sum_{n=1,3,5, \ldots}^{\infty} \frac{-1}{n} \sin (n \omega \tau)\right] B(\tau)} \\
& +B_{3}[B(\tau)]^{3}
\end{aligned}
$$

where $B_{1}$ and $B_{3}$ are explain in Eq. (57) and (59) respectively.

\subsection{Heat Transport}

The horizontally averaged Nusselt number, $\mathrm{Nu}$ is given by

$N u(\tau)=1+\frac{\left[\frac{k_{c}}{2 \pi} \int_{0}^{\frac{2 \pi}{k_{c}}}\left(\frac{\partial T_{2}}{\partial z}\right) d z\right]_{z=0}}{\left[\frac{k_{c}}{2 \pi} \int_{0}^{\frac{2 \pi}{k_{c}}}\left(\frac{\partial T_{b}}{\partial z}\right) d z\right]_{z=0}}$

After simplifying Eqs. (51), (52) and (62), we get

$N u(\tau)=1+\frac{k_{c}^{2}}{4 M \delta^{2}}[B(\tau)]^{2}$

It is clear from the above equation that the gravity modulation term $\delta_{1} \sin (\omega t)$ is present due to second order term and the next section gives details of the heat transport.

\section{RESULTS AND DISCUSSION}

In this paper, we present linear and weakly nonlinear analyses of magneto-convection in a sparsely packed porous medium under a constant vertical magnetic field with gravity modulation. For linear stability analysis, the regular perturbation method is used to calculate the correction Rayleigh number $R_{2 c}$. Weakly nonlinear analysis is performed using Ginzburg-Landau equation to investigate the effect of three different gravity modulations on heat transport. The effects of non-dimensional parameters, thermal prandtl number $\left(P r_{1}\right)$, magnetic prandtl number $\left(P r_{2}\right)$, Darcy number $(D a)$, Chandrasekhar number $(Q)$ and other porosity parameters are presented in Figs. (2)-(5). 

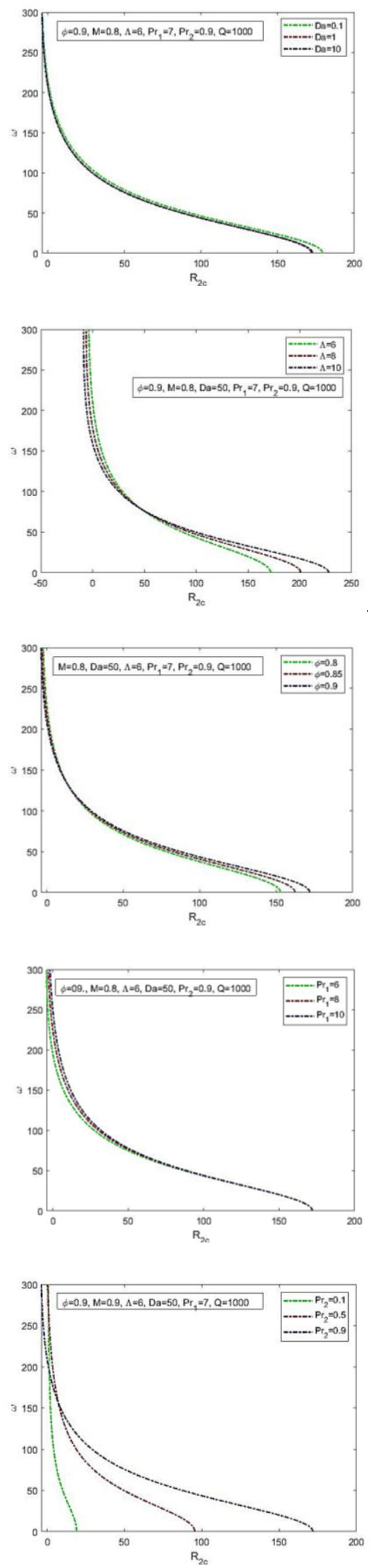

Fig. 2. Graph for correction Rayleigh number $R_{2 c}$ verses $\omega$ for different value of (a) $D a$, (b) $\Lambda$ (c) $\varphi$ (d) $P r_{1}$ and (e) $P r_{2}$.
Before we make a discussion of the results of the paper, we first mention about the physical significance of the various parameters that arise in the study. There are two Prandtl numbers, $\operatorname{Pr}_{1}$ and $\operatorname{Pr} 2$. The first one, $\operatorname{Pr}_{1}$, refers to the classical Prandtl number which is the ratio of the speeds of momentum diffusion and thermal diffusion. The Prandtl number of the liquid-saturated porous medium should be more than that of the base liquid due to the presence of the porous medium. If we consider water, $P r_{1}$ then must take a value much greater than 6. The second, $P r_{2}$, refers to the magnetic Prandtl number. This is the ratio of the viscous diffusion rate to the magnetic diffusion rate. For finitely electrically conducting liquids occupying a porous medium the parameter $\mathrm{Pr}_{2}$ usually takes values less than 1 . In the case of sun, plasma and liquid metals it takes very small values, much lesser than 1 . The other parameter that arises is the Chandrashekar number, $Q$, which is the square of the ratio of the Lorentz force to the viscous force. We consider not so small and not so large values of $Q$ in the paper signifying that the two forces are comparable. Lorentz force and viscous force oppose the flow. The latter force acts as friction whereas the former force lends rigidity to the finitely electrically conducting fluid. The parameters that correspond to the sparsely-packed porous medium are $M, \Lambda$ and $D a$ which are respectively, the ratio of heat capacities, the ratio of viscosities and the Darcy number respectively. The parameter $M$ is the ratio of the effective heat capacity of the porous medium and the heat capacity of the fluid. The effective heat capacity is larger for most fluid-saturated porous media compared to that of the fluid and hence takes a value less than 1 . The parameter $\Lambda$ is the ratio of effective viscosity of the fluid-saturated porous medium to that of the fluid. Gilver and Altobelli (1994) have shown that the range of the ratio $=\mu_{e} / \mu$ varies from 0.5 to 10.9. Compared to the effect of other parameters, $\mathrm{M}$ and $\Lambda$ have a lesser influence on the stability and the heat transport in the system. The parameter $D a$ is a geometric parameter. It involves the permeability of the porous medium and the height of the porous medium. Since we are considering a sparsely-packed porous medium the value of $D a$ is non-zero and less than 100 (Nield and Bejan 2006). Further, for a sparsely-packed porous medium the value of $\varphi$ is nearer 1 but less than 1 . Since we are considering a small-amplitude modulation in the problem the value of $\delta_{1}$ has to be small and hence we have chosen 0.1 .

Figs. 2(a)-2(e) shows the effect of modulation ( $\omega$ ) on correction Rayleigh number, $R_{2 c}$ for different parameters. The Fig. 2(a) shows that the graph of correction Rayleigh number $R_{2 c}$ verses $\omega$ for different value of Darcy number $D a$, and it decreases with increase in $D a$, thus showing the effect that increase in $D a$ destabilize the system. From the Fig. 2(b), it is observed that the correction Rayleigh number $R_{2 c}$ increases with the increase in $\Lambda$ which means that the effect of $\Lambda$ is to stabilize the system. Similar kind of results can be found in Fig. 2(c) with the increase in $\varphi$. It has been observed that from Figs. 2(d) and 2(e), the correction Rayleigh number, $R_{2 c}$ increases when the thermal prandtl number $\left(P r_{1}\right)$, 

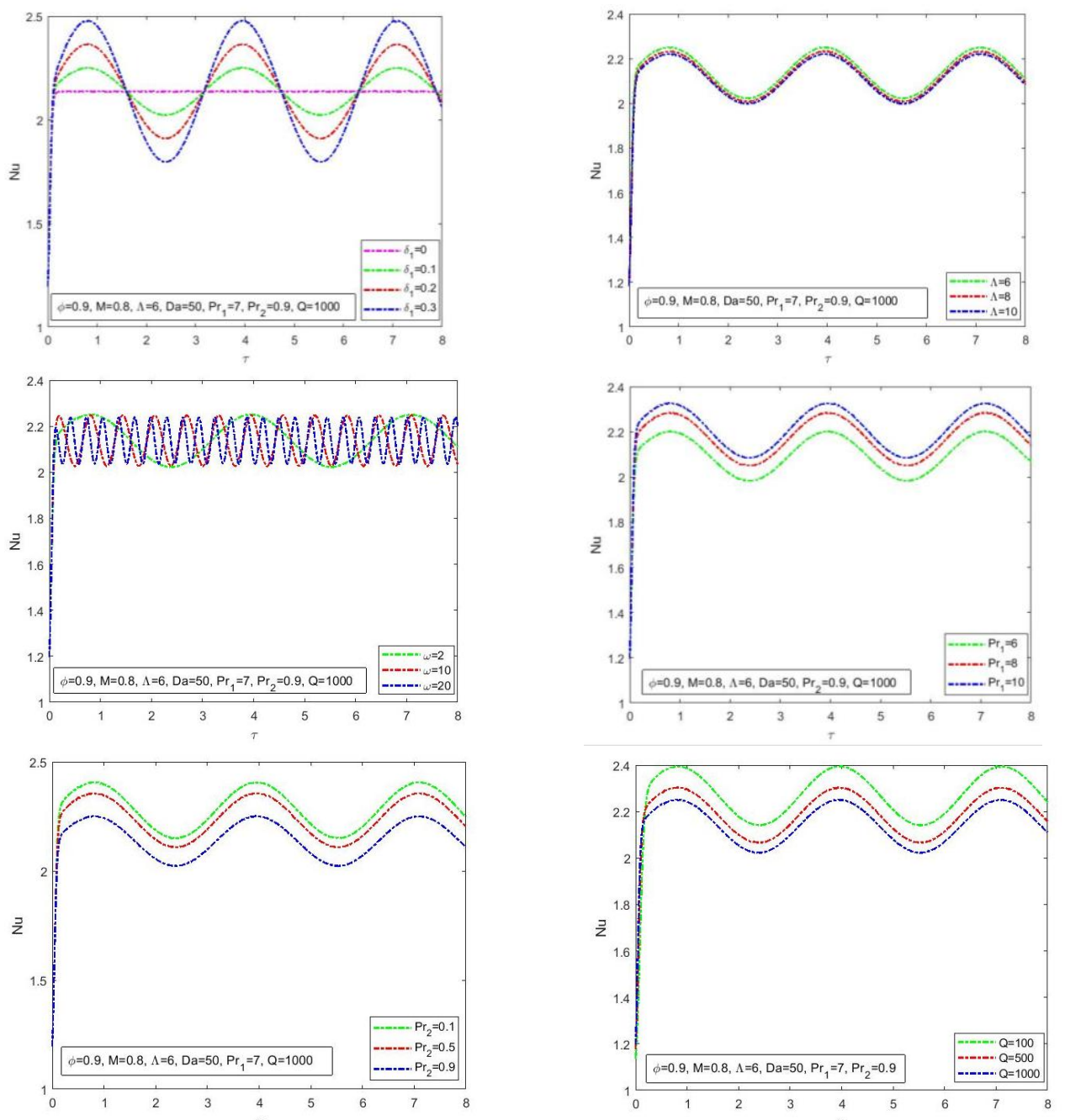

Fig. 3. Nusselt number verses $\tau$ for different value of (a) $\delta_{1}$, (b) $\Lambda$, (c) $\omega$, (d) $\operatorname{Pr}_{1}$ (d) $P r_{2}$ and (f) $Q$. for the gravity sine modulation.

magnetic prandtl number $\left(\mathrm{Pr}_{2}\right)$ increases. It means that, the effect of both parameters, thermal prandtl number $\left(P r_{1}\right)$, magnetic prandtl number $\left(P r_{2}\right)$ is to stabilize the onset of convection.

The weakly nonlinear analysis of Rayleigh-Benard convection in a sparsely packed porous medium under constant magnetic field with gravity modulation is discussed here. We have taken into account that the modulation of Rayleigh-Benard convection has been assumed to be of order $O\left(\varepsilon^{2}\right)$, that means the large amplitude of gravity is not considered. The modulated term $\left(\delta_{1} \sin (\omega \tau)\right)$ are because of the second order $O\left(\varepsilon^{2}\right)$ terms which affects the system. The influence on the heat transport due to various parameters like Chandrasekhar number $(Q)$, thermal prandtl number $\left(P r_{1}\right)$, magnetic Prandtl number $\left(P r_{2}\right)$, frequency modulation $(\omega)$ and amplitude modulation $\left(\delta_{1}\right)$ is shown in Figs. 3-5. Fig. 3(a) shows that, the increase in the amplitude of gravity modulation increases the value of Nusselt number $\mathrm{Nu}$ also. Thus the enhancement of heat transport is due to the amplitude of gravity modulation, $\delta_{1}$ and we also sketch the graph when amplitude of gravity modulation is zero and settles to a fixed value. Similar kind of observation is also found with the effect of triangular wave and square wave gravity modulation with the increase of amplitude of gravity modulation (see Figs. 4a and 5a).

From Fig. 3(b) it is evident that the increase in $\Lambda$ i.e ratio of coefficient of effective fluid viscosity to coefficient of viscosity of fluid, diminish the heat transfer. In Fig. 3(c) shows that the effect of frequency of gravity modulation on heat transport. One can easily observe that there is enhancement in heat transfer for small value of $\omega$, whereas for higher values of $\omega$ the amplitude of gravity modulation decreases and so Nusselt number Nu. If we take large values of $\omega$, the effect of gravity modulation vanish altogether, so the system is stable with the effect of $\omega$. Similar kind of observation can be found with the effect of triangular wave as well as square wave gravity modulation (see Figs. $4 \mathrm{~b}$ and $5 \mathrm{~b}$ ).

The Fig. 3(d) shows that the effect of thermal prandtl number which is nothing but ratio of kinematic viscosity by thermal diffusivity is to increase the heat transport for small value of time, and same pattern is 

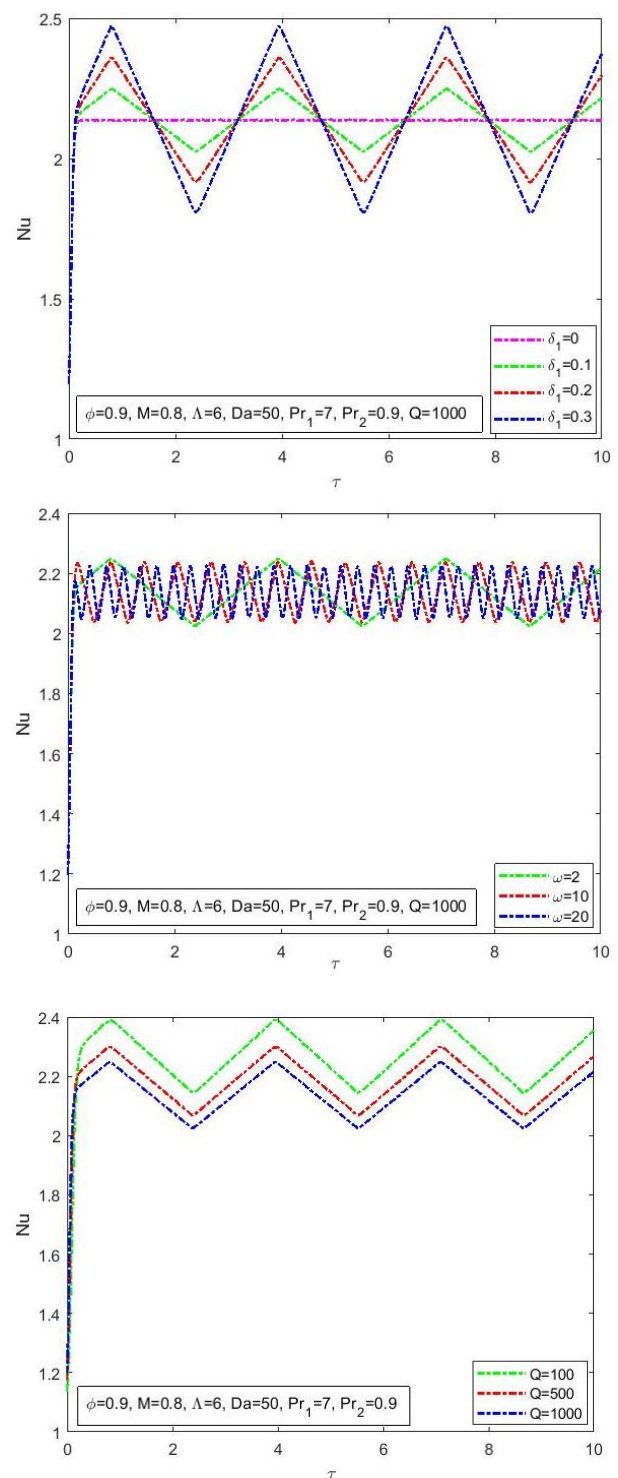

Fig. 4. Nusselt number verses $\tau$ for different value of (a) $\delta$, (b) $\omega$, (c) $Q$, for the triangular wave modulation.
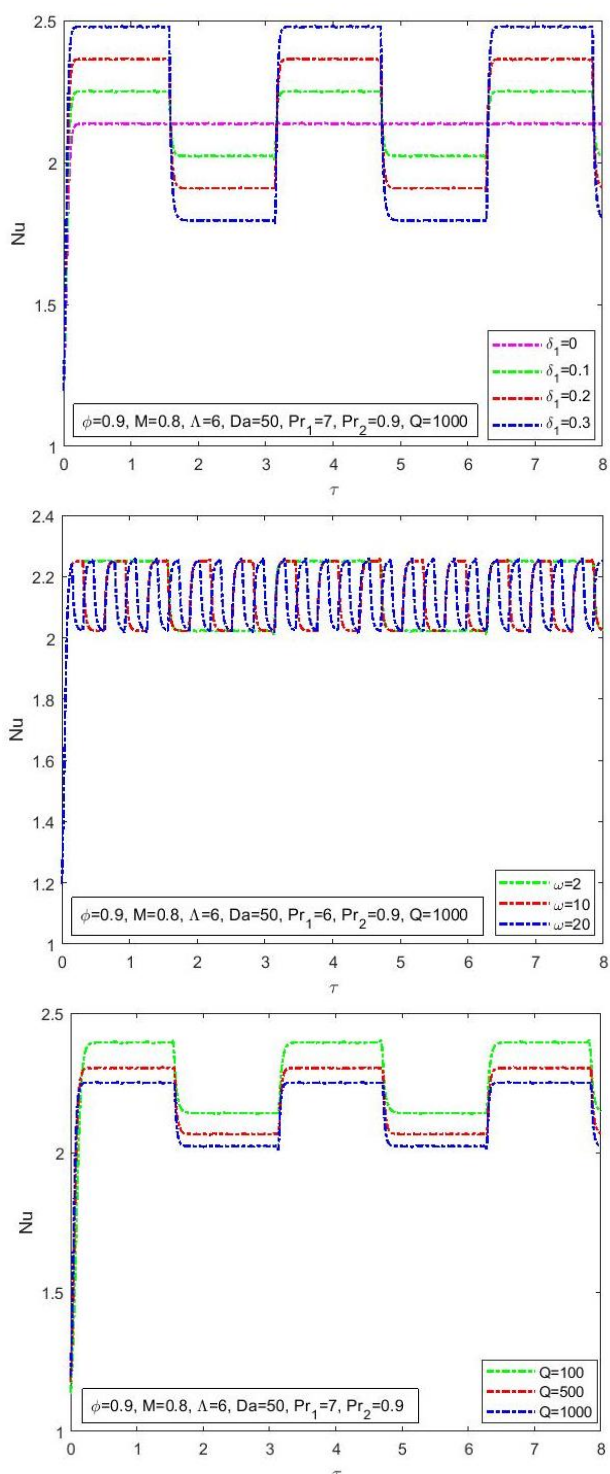

Fig. 5. Nusselt number verses $\tau$ for different value of (a) $\delta_{1}$, (b) $\omega$, (c) $Q$, for the square wave gravity modulation. also observed for large value of time also. It is also observed that when prandtl number increased then either thermal diffusivity decreased or kinematic viscosity increased. Thus results in the enhancement of heat transport in either of the cases.

Figure 3(e) shows that the effect of magnetic prandtl number $P_{2}$ which is nothing but ratio of thermal diffusivity and magnetic diffusivity is to decrease the heat transport for small value of time, i.e as magnetic prandtl number increases heat transport decreases and it is observed that magnetic prandtl number is increased then either thermal diffusivity increased or magnetic diffusivity decreased, so in both way heat transfer decreases. Same pattern are observed in case of square waves and triangular waves of gravity modulation. From Fig. 3(f) it is evident that increase in Chandrasekhar number $Q$ is diminishing the heat transfer. As Chandrasekhar number increases the Nusselt number decreases in case of square wave and triangular wave gravity modulation. Thus the effect of Chandrasekhar number is to diminish the heat transport (see Figs. $4 \mathrm{c}$ and $5 \mathrm{c}$ ) for both types of waves.

The modulation of the type that has been considered in the paper arises due to the vertical vibrations of the fluid system housed in a porous medium. The modulation gives rise to a time-dependent gravity in addition to the usual gravity (see Siddheshwar and Kanchana (2019), Siddheshwar and Meenakshi (2019) and Kanchana et al. (2020), Bhadauria and Kiran (2015)). The modulation greatly affects the onset of convection and heat transport and thus serves the purpose of regulating the dynamics in the system. 


\section{CONCLUSIONS}

1. The effect of parameters, porosity, $\varphi$, thermal prandtl number, $P r_{1}$, magnetic prandtl number, $\operatorname{Pr}_{2}$ and $\Lambda$ is to stabilize the system, whereas the effect of Darcy number, $D a$, is to destabilise the system.

2. Enhancement of the heat transport is seen due to the effect of amplitude modulation.

3. Diminished heat transport is the effect of frequency modulation is observed.

4. The effect of thermal prandtl number is to enhance the heat transport whereas the effect of magnetic prandtl number and Chandrasekhar number is to diminish the same.

5. The control of convection is a major issue in systems with fluids as a working media. This is all the more difficult if the fluid system is housed in a porous medium. The paper presents three mechanisms of controlling onset of convection and thereby the heat transfer in such fluid systems. In order the modulation effect is effective in its role, we have considered the system to be a fluid-saturated porous media.

\section{ACKNOWLEDGEMENTS}

The authors are grateful to the reviewers for their most useful comments and suggestions. The Author (RR) gratefully acknowledge the support offered by the "Science and Engineering Research Board", Department of Science \& Technology, India, under the Grant No: ECR/2017/000357 for this research work.

\section{REFERENCES}

Babu, A. B., R. Ragoju and S. G. Tagare (2011). Nonlinear Magnetoconvection in a Sparsely Packed Porous Medium, International Journal of Geophysics 207123, 1-17

Bhadauria, B. S. and A. Khan (2009). Modulated Centrifugal Convection in a Vertical Rotating Porous Layer Distant from the Axis of Rotation, Transport in porous media 79, 255-264.

Bhadauria, B. S. and P. Kiran (2013). Heat Transport in an Anisotropic Porous Medium Saturated with Variable Viscosity Liquid Un-der Temperature Modulation, Transport in porous media, 100, 279-295.

Bhadauria, B. S. and P. Kiran (2015). Weak Nonlinear Double Diffusive MagnetoConvection in a Newtonian Liquid under Gravity Modulation, Journal of Applied Fluid Mechanics, 8, 735-746.

Bhadauria, B. S., I. Hashim and P. G. Siddheshwar (2013). Study of Heat Transport in a Porous Medium Under G-jitter and Internal Heating Effects, Transport in porous media 96, 21-37.

Bhadauria, B. S., P. G. Siddheshwar and P. S. Om
(2012). Nonlinear Thermal Instability in a Rotating Viscous Fluid Layer Under Temperature/Gravity Modulation, Journal of Heat Transfer, 134, 1-9.

Bhadauria, B. S., P. G.Siddheshwar, A. K. Singh, and K. G. Vinod (2016). A Local Non-linear Stability Analysis of Modulated Double Diffusive Stationary Convection in a Couple Stress Liquid, Journal of Applied Fluid Mechanics 9, 1255-1264.

Chandrasekhar, S. (1961). Hydrodynamic and Hydromagnetic Stability, Oxford University Press, London.

Clever, R., G. Schubert and F. Busse (1993). Two dimensional oscillatory convection in a gravitationally modulated fluid layer, Journal of Fluid Mechanics 253, 663-680.

Dyko, M. P. and K. Vafai (2007). Effect of gravity modulation on convection in a horizontal annulus, International Journal of Heat and Mass Transfer 50, 348-360.

Farooq, A. and G. M. Homsy (1996). Linear and nonlinear dynamics of a differentially heated slot under gravity modulation, Journal of Fluid Mechanics, 313, 1-38

Gershuni, G. Z., E. M. Zhukhovitskii and I. S. Iurkov (1970). On convective stability in the presence of periodically varying parameter, Journal of Applied Mathematics and Mechanics 34, 442452 .

Givler, R. C. and S. A. Altobelli (1994). A determination of the effective viscosity for the Brinkman-Forchheimer flow model, Journal of Fluid Mechanics 258, 355-370.

Gresho, P. M. and R. L. Sani (1970). The effect of gravity modulation on the stability of a heated fluid layer, Journal of Fluid Mechanics 40, 783 806.

Kanchana, C., P. G. Siddheshwar and Y. Zhao (2020). Regulation of heat transfer in RayleighBenard convection in Newtonian liquids and Newtonian nanoliquids using gravity, boundary temperature and rotational modulations. Journal of Thermal Analysis and Calorimetry.

Malashetty, M. S. and Begum I. (2011). Effect of Thermal/Gravity Modulation on the On-set of Convection in a Maxwell Fluid Saturated Porous Layer, Transport in porous media 90, 889-909.

Malashetty, M. S. and M. S. Swamy (2011). Effect of gravity modulation on the onset of thermal convection in rotating fluid and porous layer, Physics of Fluids 23, 064108

Malashetty, M. S. and V. Padmavathi (1997). Effect of gravity modulation on the onset of convection in fluid and porous layer, International Journal of Engineering Science 35, 829-840.

Malkus, W. V. R and G. Veronis (1958). Finite amplitude cellular convection. Journal of Fluid Mechanics 4, 225-260. 
R. Ragoju and S. Shekhar / JAFM, Vol. 13, No. 6, pp. 1937-1947, 2020.

Nield, D. A. and A. Bejan (2006) Convection in porous media, 3rd ed., Springer, New York.

Ravi, R., C. Kanchana, G. J. Reddy and H. Basha (2018). Study of Soret and Dufour effects and secondary instabilities on Rayleigh-Benard convection in a couple stress fluid, The European Physical Journal Plus 133, 513-532.

Shivakumara, I. S., J. Lee, M. S. Malashetty and S. Sureshkumar (2011). Effect of Thermal Modulation on the Onset of Convection in Walters B Viscoelastic Fluid-Saturated Porous Medium, Transport in porous media 87, 291307.

Siddheshwar, P. G. (2010). A Series Solution for the Ginzburg-Landau Equation with a TimePeriodic Coefficient. Applied Mathematics 3, 542-554.

Siddheshwar, P. G. and C. Kanchana (2019). Effect of trigonometric sine, square and triangular wave-type time-periodic gravity-aligned oscillations on RayleighBnard convection in Newtonian liquids and Newtonian nanoliquids. Meccanica 54, 451469.

Siddheshwar, P. G. and N. Meenakshi (2019). Comparison of the effects of three types of timeperiodic body force on linear and non-linear stability of convection in nano-liquids, European Journal of Mechancis, B/Fluids 77, 221-229.

Siddheshwar, P. G., B. S. Bhadauria, P. Mishra and A. K. Srivastava (2011). Study of heat transport by stationary magneto-convection in a Newtonian liquid under temperature or gravity modulation using Ginzburg-Landau mode, International Journal of Non-Linear Mechanics 47, 418-425.

Venezian, G. I. (1969). Effect of modulation on the onset of thermal convection, Journal of Fluid Mechanics 35, 243-254. 